\title{
DETERMINACIÓN DE LA CARGA PERMANENTE CORRESPONDIENTE A DISTINTOS TIPOS DE MAMPOSTERÍAS EN EDIFICACIONES DE VIVIENDA
}

\section{DETERMINATION OF THE PERMANENT LOAD DUE TO DIFFERENT TYPES OF MASONRY IN HOUSING BUILDINGS}

ESTUARDO PÁEZ ESPINOSA ${ }^{1}$

CARLOS ANDRÉS PÁEZ VARGAS ${ }^{2}$

Recibido: 25 de octubre de 2017 Aceptado: 29 enero de 2018

\footnotetext{
${ }_{1}^{1}$ Pontificia Universidad Católica del Ecuador, Facultad de Ingeniería, Quito Ecuador (epaeze@puce.edu.ec) 2 University of Illinois at Urbana-Champaign, Urbana, Illinois, USA (carlosp2@illinois.edu),
} 
illil 142 


\section{DETERMINACIÓN DE LA CARGA PERMANENTE CORRESPONDIENTE A DISTINTOS TIPOS DE MAMPOSTERÍAS EN EDIFICACIONES DE VIVIENDA}

\section{DETERMINATION OF THE PERMANENT LOAD DUE TO DIFFERENT TYPES OF MASONRY IN HOUSING BUILDINGS}

Estuardo Páez Espinosa, Carlos Andrés Páez Vargas

Palabras clave: Carga permanente, paredes, peso propio, análisis estructural, cargas, mamposterías.

Keywords: Permanent loads, walls, self-weight, structural analysis, loads, masonry.

\section{RESUMEN}

Varios códigos de construcción no establecen el valor de cargas permanentes debidas al peso de paredes de mampostería que deben considerarse en el análisis estructural de edificaciones de vivienda. En el presente artículo se presentan valores que pueden ser in- cluidos en el análisis por parte del calculista; entre ellos, la densidad de paredes esperada para edificaciones de vivienda, así como también la carga por unidad de superficie que se sugiere debe ser considerada, en función del tipo de materiales que intervienen en la construcción. 


\begin{abstract}
Several building codes do not establish the value of the permanent loads due to the weight of masonry walls that should be considered for the structural analysis of housing buildings. This article presents the required parameters for the

computation of this type of load such as the expected density of walls for housing buildings as well as the load per surface unit which is suggested to be considered according to the type of materials used during construction.
\end{abstract}

\title{
INTRODUCCIÓN
}

Para todo diseñador estructural es imprescindible la correcta estimación de las cargas que actuarán en una edificación a lo largo de toda su vida útil. Se deben incluir las cargas relacionadas con los pesos propios de todos los materiales que componen la estructura misma; de aquellos que estarán presentes permanentemente en la estructura, otras solicitaciones asociadas al uso (denominadas cargas vivas); otras relacionadas con la interacción estructura-medio (dentro de este caso, cargas sísmicas, viento, presiones hidrostáticas, sobrecargas por granizo, nieve, ceniza volcánica, etc) y otras provenientes del uso al que se destinará la estructura, tales como cargas de frenado, fuerzas centrífugas, fuerzas de impacto, etc.

Dentro de las cargas permanentes se encuentra el peso de las mampos- terías, las cuales son parte integrante de los elementos no estructurales de edificaciones de vivienda, aulas, edificios de oficinas, teatros, auditorios, etc. La mayoría de los códigos de construcción actualmente en vigencia no establecen el valor de carga por $\mathrm{m}^{2}$ de superficie correspondiente a paredes de mamposterías para viviendas. Solamente algunos códigos lo hacen, como es el caso del código español denominado "Seguridad Estructural Acciones en la Edificación SE-AE" ${ }^{\prime \prime}$ que en su sección segunda establece:

"En el caso de tabiques ordinarios cuyo peso por metro cuadrado no sea superior a $1,2 \mathrm{kN} / \mathrm{m}^{2}$ y cuya distribución en planta sea sensiblemente homogénea, su peso propio podrá asimilarse a una carga equivalente

${ }^{1}$ Dirección General de Arquitectura, Vivienda y Suelo; Seguridad Estructural Acciones en la Edificación SEAC, Madrid, Autor, 2009, pág. 3 
uniformemente distribuida. Como valor de dicha carga equivalente se podrá adoptar el valor del peso por metro cuadrado de alzado multiplicado por la razón entre la superficie de tabiquería y la de la planta considerada." "En el caso de tabiquería más pesada, esta podrá asimilarse al mismo valor de carga equivalente uniforme citado más un incremento local, de valor igual al exceso de peso del tabique respecto a 1,2 kN por $\mathrm{m}^{2}$ de alzado. En general, en viviendas bastará considerar como peso propio de la tabiquería una carga de 1,0 kN por cada $\mathrm{m}^{2}$ de superficie construida"

La Norma Ecuatoriana dela Construcción (NEC) define como cargas permanentes lo siguiente:

"Las cargas permanentes (o cargas muertas) están constituidas por los pesos de todos los elementos estructurales, tales como: muros, paredes, recubrimientos, instalaciones sanitarias, eléctricas, mecánicas, máquinas y todo artefacto integrado permanentemente a la estructura."

"Las cargas permanentes serán tomadas en cuenta de acuerdo con lo expuesto en el apéndice 4. Para los elementos no encontrados en el apéndice 4, se deberá describir y justificar los datos elegidos en la memoria de cálculo." 2

En el apéndice 4 de la Norma Ecuatoriana de la construcción únicamente se establece un peso unitario de $12 \mathrm{kN} / \mathrm{m}^{3}$ para bloques huecos de hormigón, y $8.5 \mathrm{kN} / \mathrm{m}^{3}$ para bloques huecos de hormigón alivianados ${ }^{3}$

Se observa una falta de información en lo referente a la determinación de la carga permanente correspondiente a paredes de mampostería, que debe aplicarse en el diseño estructural.

El objetivo del presente estudio es determinar el valor de la carga permanente correspondiente a distintas densidades de paredes elaboradas con diferentes materiales de construcción en edificaciones de vivienda.

Además, se busca :

- Determinar un valor de carga permanente correspondiente a mamposterías de bloque de 10, 15 y 20 cm, con enlucidos a ambos lados.

- Determinar un valor de carga permanente correspondiente a mamposterías de ladrillo, con enlucidos a ambos lados.

\footnotetext{
${ }^{2}$ Comité Ejecutivo de la Cámara de la Construcción, Norma Ecuatoriana de la Construcción NEC; NEC-SGCG Cargas no sísmicas, Quito, Autor, pág 11

${ }^{3}$ Ibíd; pág. 22
} 
- Determinar el valor correspondiente a carga permanente, de acuerdo con diferentes concentraciones, o "densidades" de paredes en edificaciones destinadas a viviendas.

\section{METODOLOGÍA}

La Norma Española ${ }^{4}$ establece la metodología a seguir para establecer el peso de mamposterías por metro cuadrado, la misma que se aplicó en la presente investigación y que consiste en lo siguiente:

Se realizó un muestreo de materiales comúnmente utilizados en nuestro medio para la elaboración de mamposterías: bloque de hormigón y ladrillo. De cada uno de ellos se obtuvo el peso unitario y las dimensiones.

Paralelamente se elaboraron probetas de mortero con diferentes relaciones volumétricas de arena cemento, empleando también los materiales más comúnmente utilizados en el Distrito Metropolitano de Quito. Se obtuvieron los pesos unitarios correspondientes.

Ade'm ás, se analizaron varios diseños arquitectónicos de viviendas, con el fin de obtener la densidad de paredes por cada metro cuadrado de superficie de construcción, es decir, la relación entre la superficie vertical de paredes y la superficie en planta de la edificación.

A partir de la observación del proceso constructivo de paredes en la industria de la construcción dentro del Distrito Metropolitano, se determinó el procedimiento para construcción de paredes de bloque y de ladrillo, que a su vez permitió la obtención del peso por $\mathrm{m}^{2}$ de mamposterías con espesores de 10, 15 y $20 \mathrm{~cm}$ en el caso de bloques y el peso por $\mathrm{m}^{2}$ de paredes de ladrillo, denominado "mambrón" en nuestro medio. En los dos casos, bloque y ladrillo, se consideró un enlucido de 1,5 cm de espesor en las dos caras de la pared.

Con los datos obtenidos se calculó la carga debida al peso por $\mathrm{m}^{2}$ de mamposterías por cada $\mathrm{m}^{2}$ de construcción en viviendas.

Los valores obtenidos son aplicables a edificaciones de vivienda dentro del Distrito Metropolitano de Quito. El procedimiento descrito en el presente artículo podría extrapolarse para realizar la determinación de cargas de este tipo en otros lugares del país, considerando las características de los materiales y los sistemas constructivos en cada localización geográfica.

\footnotetext{
${ }^{4}$ Dirección General de Arquitectura, Vivienda y Suelo; op. cit, supra.,nota 1, pág. 3.
} 


\section{MATERIALES}

Se analizaron los materiales comúnmente utilizados para la construcción de mamposterías:

- Bloque alivianado de $20 \times 40 \times 10 \mathrm{~cm}$

- Bloque alivianado de $20 \times 40 \times 15 \mathrm{~cm}$

- Bloque de carga de $20 \times 40 \times 10 \mathrm{~cm}$

- Bloque de carga de $20 \times 20 \times 15 \mathrm{~cm}$

- Bloque de carga de $20 \times 20 \times 20 \mathrm{~cm}$

- Ladrillo mambrón

- Mortero con dosificación volumétrica 1:3

- Mortero con dosificación volumétrica 1:4

- Mortero con dosificación volumétrica 1:5

Para la determinación del peso unitario de los bloques de hormigón y ladrillos, se obtuvieron 250 y 70 muestras, respectivamente, en diferentes centros de distribución de materiales de construcción en el Distrito Metropolitano de
Quito. El número de muestras escogido para cada tipo de unidad de mampostería fue determinado con el fin de realizar un estudio con significancia estadística.

Se ha tomado especial cuidado en que los bloques no se hallen saturados pues esta es una condición que no se produce en la ocupación final de la edificación. Los bloques obtenidos se ensayaron con un grado de humedad similar al que tendrían en la pared seca, una vez construida y lista para cumplir su función.

En el caso de los morteros, se han realizado en el laboratorio 12 probetas para cada dosificación anteriormente señalada.

Como resultado del proceso de medición y pesaje se obtuvieron los siguientes valores:

\begin{tabular}{|l|r|r|r|r|r|r|r|}
\hline $\begin{array}{l}\text { Unidad de mam- } \\
\text { postería }\end{array}$ & \multicolumn{1}{|c|}{$\begin{array}{c}\text { Ancho } \\
\text { nominal }(\mathrm{cm})\end{array}$} & Peso $(\mathrm{kg})$ & $\begin{array}{c}\text { Largo } \\
(\mathrm{cm})\end{array}$ & $\begin{array}{c}\text { Profundidad } \\
(\mathrm{cm})\end{array}$ & $\begin{array}{c}\text { Ancho } \\
(\mathrm{cm})\end{array}$ & $\begin{array}{c}\text { Volumen } \\
\left(\mathrm{cm}^{3}\right)\end{array}$ & $\begin{array}{c}\text { P. unitario } \\
\left(\mathrm{kg} / \mathrm{m}^{3}\right)\end{array}$ \\
\hline \multirow{3}{*}{ Bloque de carga } & 10 & 8,44 & 40,1 & 19,7 & 10,0 & 7904,6 & 1068,52 \\
\cline { 2 - 8 } & 15 & 10,94 & 40,1 & 19,8 & 15,0 & 11918,0 & 917,39 \\
\hline \multirow{2}{*}{ Bloque alivianado } & 10 & 14,12 & 40,1 & 19,6 & 20,0 & 15671,1 & 901,06 \\
\cline { 2 - 8 } & 10 & 4,65 & 38,4 & 18,2 & 8,5 & 5943,4 & 783,18 \\
\hline Ladrillo mambrón & 15 & 6,30 & 38,3 & 18,0 & 13,9 & 9619,1 & 655,23 \\
\hline
\end{tabular}

Tabla 1. Dimensiones y peso unitario de unidades de mampostería 
En la Norma Ecuatoriana de la Construcción (NEC), NEC-SE-CG, apéndice 4 , tabla 8,5 se encuentran valores referenciales de los pesos unitarios de los materiales de construcción más utilizados. En esa tabla se especifica un valor de peso unitario de $12 \mathrm{kN} / \mathrm{m}^{3}$ para bloque hueco de hormigón y $8.5 \mathrm{kN} / \mathrm{m}^{3}$ para bloque hueco de hormigón alivianado

Los pesos unitarios de bloques de hormigón alivianados determinados a base de la muestra, se encuentran entre 9.0 y $10.7 \mathrm{kN} / \mathrm{m}^{3}$ los cuales son inferiores al valor referencial establecido en la norma $\left(12 \mathrm{kN} / \mathrm{m}^{3}\right)$

En cuanto al bloque alivianado, los valores de peso unitario se encuentran entre 6.6 y $7.8 \mathrm{Kg} / \mathrm{m}^{3}$, los cuales también son inferiores al valor establecido en la norma ecuatoriana $\left(8.5 \mathrm{kN} / \mathrm{m}^{3}\right)$.
Los materiales empleados para elaborar morteros son: cemento Portland tipo 1P, y tres tipos de arena, comúnmente utilizados en el Distrito Metropolitano de Quito:

- Arena de río obtenida de las minas del río Guayllabamba

- Arena fina, obtenida de las minas de la Mitad del Mundo, (San Antonio de Pichincha)

- Polvo amarillo, agregado fino obtenido también de las minas de la Mitad del Mundo. (San Antonio de Pichincha)

Del pesaje de las probetas realizadas, se obtuvieron los siguientes resultados:

\begin{tabular}{|l|l|r|r|r|}
\cline { 2 - 5 } \multicolumn{1}{c|}{} & Dosificación & $1: 3$ & $1: 4$ & $1: 5$ \\
\hline Arena amarilla & Peso unitario $\left(\mathrm{kN} / \mathrm{m}^{3}\right)$ & 16,75 & 16,12 & 15,77 \\
\hline Arena fina & Peso unitario $\left(\mathrm{kN} / \mathrm{m}^{3}\right)$ & 17,78 & 16,80 & 16,57 \\
\hline Arena de Guayllabamba & Peso unitario $\left(\mathrm{kN} / \mathrm{m}^{3}\right)$ & 18,27 & 17,64 & 17,48 \\
\hline
\end{tabular}

Tabla 2. Densidad de morteros de arena y cemento

La Norma Ecuatoriana de la unitario de $20 \mathrm{kN} / \mathrm{m} 3$ para el mortero Construcción NEC'11 establece un peso compuesto por arena y cemento. ${ }^{6}$ Los

\footnotetext{
${ }^{5}$ Comité Ejecutivo de la Cámara de la Construcción, Norma Ecuatoriana de la Construcción NEC; NEC-SGCG Cargas no sísmicas, Quito, Autor, pág 22

${ }^{6}$ Comité Ejecutivo de la Cámara de la Construcción, Norma Ecuatoriana de la Construcción NEC; NEC-SGCG Cargas no sísmicas, Quito, Autor, pág 22
} 
valores obtenidos en laboratorio presentan diferencias significativas siempre en defecto, con relación a la señalada en la Norma Ecuatoriana de la Construcción.

En cuanto a las mamposterías mismas, se consideraron unidades de análisis representativas para cada tipo de bloque y espesor de pared, en las que se ha tomado en cuenta las cantidades de materiales necesarios, tomadas directamente de la observación de la práctica constructiva.
Se consideró además enlucidos por ambos lados de la mampostería, con un espesor de $1.5 \mathrm{~cm}$ en cada uno de ellos.

Luego de aplicar los pesos unitarios promedio de cada uno de los materiales se obtuvieron los siguientes pesos por metro cuadrado de mampostería. Se ha considerado una dosificación de mortero arena - cemento 1:3 para unión de bloques y una dosificación 1:4 para enlucido:

\begin{tabular}{|c|c|c|c|c|c|c|c|c|}
\hline \\
\hline & & & \multicolumn{3}{|c|}{ Bloque prensado } & \multicolumn{2}{|c|}{ Bloque alivianado } & Ladrillo \\
\hline \multicolumn{3}{|l|}{ Ancho nominal (cm) } & 10 & 15 & 20 & 10 & 15 & 8 \\
\hline \multicolumn{3}{|c|}{ Número de mampuestos (por m²) } & 11,34 & 11,34 & 11,34 & 12,79 & 12,79 & 30,36 \\
\hline \multicolumn{3}{|c|}{ Peso individual del mampuesto $(\mathrm{kg})$} & 8,44 & 10,94 & 14,12 & 4,65 & 6,30 & 6,23 \\
\hline \multicolumn{3}{|l|}{ Mortero de unión $\left(\mathrm{m}^{3}\right)$} & 0,0246 & 0,0369 & 0,0492 & 0,0225 & 0,0367 & 0,0315 \\
\hline \multicolumn{3}{|c|}{ Mortero de enlucido $\left(\mathrm{m}^{3}\right)$} & 0,0400 & 0,0400 & 0,0400 & 0,0400 & 0,0400 & 0,0400 \\
\hline \multicolumn{3}{|l|}{ Espesor de pared (m) } & 0,140 & 0,190 & 0,240 & 0,125 & 0,179 & 0,199 \\
\hline Mortero & Dosificación & Densidad $\left(\mathrm{kg} / \mathrm{m}^{3}\right)$ & \multicolumn{6}{|c|}{ Peso por $\mathrm{m}^{2}$ de pared de mampostería $\left(\mathrm{kg} / \mathrm{m}^{2}\right)$} \\
\hline \multirow{2}{*}{ Arena de Guayllabamba } & $1: 3$ & 1827,03 & \multirow{2}{*}{211,18} & \multirow{2}{*}{261,93} & \multirow{2}{*}{320,44} & \multirow{2}{*}{171,13} & \multirow{2}{*}{218,28} & \multirow{2}{*}{317,29} \\
\hline & $1: 4$ & 1764,28 & & & & & & \\
\hline \multirow{2}{*}{ Arena fina } & $1: 3$ & 1778,00 & \multirow{2}{*}{206,62} & \multirow{2}{*}{256,77} & \multirow{2}{*}{314,68} & \multirow{2}{*}{166,68} & \multirow{2}{*}{213,13} & \multirow{2}{*}{312,39} \\
\hline & $1: 4$ & 1680,46 & & & & & & \\
\hline \multirow{2}{*}{ Polvo amarillo } & $1: 3$ & 1675,20 & \multirow{2}{*}{201,39} & \multirow{2}{*}{250,28} & \multirow{2}{*}{306,92} & \multirow{2}{*}{161,66} & \multirow{2}{*}{206,64} & \multirow{2}{*}{306,44} \\
\hline & $1: 4$ & 1612,82 & & & & & & \\
\hline \multicolumn{3}{|c|}{ Valor máximo de peso por $\mathrm{m}^{2}\left(\mathrm{~kg} / \mathrm{m}^{2}\right)$} & 211,18 & 261,93 & 320,44 & 171,13 & 218,28 & 317,29 \\
\hline
\end{tabular}

Tabla 3. Peso por $\mathrm{m}^{2}$ de paredes de mampostería 


\section{DENSIDAD DE PAREDES DE MAMPOSTERÍA}

Se define como tal la relación entre la superficie vertical de paredes de mampostería y la superficie en planta de la edificación. Esta relación da como resultado el número de metros cuadrados de mampostería por cada metro cuadrado de superficie bruta de construcción.

Se seleccionaron 50 planos de vivienda, de diferentes superficies, con el fin de determinar la cantidad de metros cuadrados de paredes que se encuentran distribuidos en la superficie en planta de la vivienda. ( $\mathrm{m}^{2}$ de mamposterías / $\mathrm{m}^{2}$ de superficie en planta).

Se ha considerado una altura de entrepiso típica de $2.40 \mathrm{~m}$, la cual es comúnmente utilizada para el tipo de vivienda analizado. Los resultados obtenidos son los siguientes:

\begin{tabular}{|l|r|}
\hline \multicolumn{2}{|l|}{ Densidades de paredes de mampostería } \\
\hline Valor promedio $\left(\mathrm{m}^{2} / \mathrm{m}^{2}\right)$ & 1,591 \\
\hline Valor máximo $\left(\mathrm{m}^{2} / \mathrm{m}^{2}\right)$ & 2,075 \\
\hline Valor mínimo $\left(\mathrm{m}^{2} / \mathrm{m}^{2}\right)$ & 1,185 \\
\hline
\end{tabular}

Tabla 4. Densidades de paredes de mampostería

El valor máximo de $2.075 \mathrm{~m}^{2} / \mathrm{m}^{2}$ corresponde a viviendas con alta densidad de paredes, mientras que el valor mínimo de $1.185 \mathrm{~m}^{2} / \mathrm{m}^{2}$ se halla en viviendas con baja densidad, es decir, en aquellas que poseen ambientes más amplios, las cuales se encuentran en proyectos orientados generalmente hacia un estrato socioeconómico medio alto $y$ alto.

A continuación, se multiplicó el peso por $\mathrm{m}^{2}$ de la mampostería por la densidad de paredes en la planta arquitectónica. Se obtuvieron los siguientes valores: 


\begin{tabular}{|c|c|c|c|c|c|c|c|}
\cline { 2 - 8 } \multicolumn{2}{c|}{} & \multicolumn{3}{l|}{ Bloque prensado } & \multicolumn{2}{l|}{ Bloque alivianado } & Ladrillo \\
\hline \multicolumn{2}{|c|}{ Ancho $(\mathrm{cm})$} & 10 & 15 & 20 & 10 & 15 & 8 \\
\hline \multicolumn{2}{|c|}{ Peso por m ${ }^{2}$ de paredes $\left(\mathrm{kg} / \mathrm{m}^{2}\right)$} & 211,18 & 261,93 & 320,44 & 171,13 & 218,28 & 317,29 \\
\hline \multicolumn{2}{|c|}{ Densidad $\left(\mathrm{m}^{2} / \mathrm{m}^{2}\right)$} & \multicolumn{6}{c|}{ Carga por unidad de superficie $\left(\mathrm{kg} / \mathrm{m}^{2}\right)$} \\
\hline Valor promedio & 1,591 & 336,07 & 416,84 & 509,94 & 272,34 & 347,37 & 504,92 \\
\hline Valor máximo & 2,075 & 438,25 & 543,57 & 664,99 & 355,15 & 452,98 & 658,45 \\
\hline Valor mínimo & 1,185 & 250,31 & 310,47 & 379,82 & 202,85 & 258,73 & 376,08 \\
\hline
\end{tabular}

Tabla 5. Carga por unidad de superficie de paredes de mampostería

\section{CONCLUSIONES Y RECOMENDACIONES}

Al no disponer de una referencia en la Norma Ecuatoriana de la Construcción (NEC11), muchos diseñadores estructurales aplican criterios no sustentados o tomados de otras realidades para considerar el peso de las paredes de mampostería en las edificaciones. Uno de los criterios era tomar el mismo valor de la carga viva ( $2 \mathrm{kN} / \mathrm{m} 2$ para viviendas en la norma ecuatoriana), equivalentes únicamente a mamposterías de bloque alivianado de $10 \mathrm{~cm}$.
La norma española (una de las pocas normas que establecen recomendaciones para este valor), establece que:

"En general, en viviendas bastará considerar como peso propio de la tabiquería una carga de 1,0 kN por cada $\mathrm{m}^{2}$ de superficie construida"7

Lo cual implicaría utilizar cargas inferiores hasta en un $60 \%$ con relación a las obtenidas en el presente estudio.

Consecuentemente a lo expuesto, se recomienda utilizar los siguientes valores:

\footnotetext{
${ }^{7}$ Dirección general de Arquitectura, Vivienda y Suelo; Seguridad Estructural Acciones en la Edificación SEAC, Madrid, Autor, 2009, pág. 3
} 


\begin{tabular}{|c|c|c|c|c|c|c|c|}
\hline & & Bloque & nsado & & Bloque & nado & Ladrillo \\
\hline \multicolumn{2}{|c|}{ Ancho nominal $(\mathrm{cm})$} & 10 & 15 & 20 & 10 & 15 & 8 \\
\hline \multicolumn{2}{|c|}{ Densidad paredes $\left(\mathrm{m}^{2} / \mathrm{m}^{2}\right)$} & \multicolumn{6}{|c|}{ Carga por unidad de superficie kg/m2 } \\
\hline Media & 1,591 & 336,07 & 416,84 & 509,94 & 272,34 & 347,37 & 504,92 \\
\hline Alta & 2,075 & 438,25 & 543,57 & 664,99 & 355,15 & 452,98 & 658,45 \\
\hline Baja & 1,185 & 250,31 & 310,47 & 379,82 & 202,85 & 258,73 & 376,08 \\
\hline
\end{tabular}

Tabla 6. Valores recomendados de carga por unidad de superficie de paredes de mampostería

Los valores que aparecen en la tabla anterior muestran que, en el caso del bloque de carga de $20 \mathrm{~cm}$ de ancho nominal, los valores de carga permanente correspondientes (considerando una densidad de paredes correspondiente a $2.075 \mathrm{~kg} / \mathrm{m}^{2}$ verticales de pared por $\mathrm{m}^{2}$ de superficie bruta de construcción) pueden alcanzar valores de hasta $665 \mathrm{Kg} / \mathrm{m}^{3}$.

En el caso del ladrillo mambrón, los valores pueden alcanzar hasta
$658 \mathrm{~kg} / \mathrm{m}^{2}$ con una alta densidad de paredes.

En todos los casos, la altura de entrepiso adoptada es de 2.40 m, la más comúnmente utilizada para fines de vivienda. Sin embargo, debe tenerse presente que los ambientes más amplios requieren una mayor altura de entrepiso, y viceversa, cuando los ambientes son pequeños, es necesaria una menor altura de entrepiso para guardar las debidas proporciones de los ambientes. 


\section{BIBLIOGRAFÎA}

Instituto Nacional de Tecnología Industrial. Centro de Investigación de los Reglamentos Nacionales de Seguridad para las Obras Civiles. (2005). Reglamento Argentino de Cargas Permanentes y Sobrecargas Mínimas de Diseño para Edificios y otras Estructuras, Buenos Aires.

ABNT-Associação Brasileira de Normas Técnicas. Comitê Brasileiro de Construção Civil. (1980). Cargas para o cálculo de estruturas de edificações, Rio de Janeiro.

Instituto Nacional de Normalización. (2009). Diseño estructural - Cargas permanentes y cargas de uso, Santiago de Chile: s/e.

Asociación Colombiana de Ingeniería Sísmica. Comisión Asesora Permanente para el Comité Ejecutivo de la Norma Ecuatoriana de la Construcción. (2014). Norma Ecuatoriana de la construcción, NEC-SE-CG, Quito: s/e.
Dirección General de Arquitectura, Vivienda y Suelo. (2009). Seguridad Estructural Acciones en la Edificación, Madrid: s/e.

American Society of Civil Engineers. (2010). Minimum Design Loads for Buildings and Other Structures ASCE Standard ASCE SEl 7-10, Virginia: s/e.

Asamblea Legislativa del Distrito Federal. (2004). Reglamento de Construcciones para el Distrito Federal, México D.F.: s/e.

Instituto de la Construcción y Gerencia. (2006). Norma E.020 Cargas, Lima: s/e.

Dirección General Sectorial de Edificaciones. (1988). Criterios y Acciones Mínimas para el Proyecto de Edificaciones, Caracas, Comisión Venezolana de Normas Industriales: s/e. 
